\title{
Global specialists talk on latest advances in cough studies: a close-up of the Third International Cough Conference in Guangzhou, China
}

\author{
Wei Luo, Tingting Xu, Yuehan Chen, Ziyu Jiang, Wenzhi Zhan, Li Long, Kefang Lai \\ State Key Laboratory of Respiratory Disease, National Clinical Research Center for Respiratory Disease, Guangzhou Institute of Respiratory \\ Health, the First Affiliated Hospital of Guangzhou Medical University, Guangzhou, Guangdong 510120, China \\ Correspondence to: Kefang Lai. State Key Laboratory of Respiratory Disease, National Clinical Research Center for Respiratory Disease, Guangzhou \\ Institute of Respiratory Health, the First Affiliated Hospital of Guangzhou Medical University, Guangzhou, Guangdong 510120, China. \\ Email: klai@163.com.
}

Submitted Dec 03, 2019. Accepted for publication Dec 18, 2019.

doi: $10.21037 /$ jtd.2019.12.90

View this article at: http://dx.doi.org/10.21037/jtd.2019.12.90

The $3^{\text {rd }}$ International Cough Conference (ICC) was held on November 7-9, 2019, attended by over 500 participants from China and other countries worldwide, and chaired by Prof. Nanshan Zhong, Prof. Kefang Lai, Prof. Kianfan Chung and Prof. Peter Dicpinigaitis (Figure 1).

More than 30 globally reputed cough specialists from Britain, the United States, Japan, Korea, Denmark, Australia and China showed up in the event. The conference incorporated key-note presentations and poster sessions to cover the epidemiology and pathogenesis, cough hypersensitivity, evaluation, diagnosis and therapy for common and uncommon etiologies, future direction for chronic cough study, offering a platform for young scientists and clinicians to share their work, as well as a frontier and practical academic feast for all attendees (Figures 2,3).

The hot topics are listed as follows.

\section{Session 1: Regulation of chronic cough}

Peripheral and central mechanism of cough hypersensitivity

\section{Speaker: Stuart B. Mazzone}

Airway inflammation contributes to peripheral sensitization through the activation and sensitization of vagal sensory nerve terminals. Environmental exposures can sensitize different sensory nerve terminals, causing the heterogeneity in peripheral pathways. Enhanced midbrain activity, as well as reduced cortex inhibitory control in cough hypersensitive patients has been found by fMRI, suggesting that central plasticity also involve in cough hypersensitivity. The complexity in central plasticity therefore forms the basis of the heterogeneity in cough hypersensitivity.

\section{Airway bypersensitivity and cough hyperresponsiveness} induced by eosinophil granule-derived cationic proteins

Speaker: Lu-Yuan Lee

Eosinophil cationic proteins can exert a direct, charge-dependent and long-lasting sensitizing effect on bronchopulmonary C-fiber afferents. The sensitizing effect is resulted from a positive interaction with TRPV1 receptor, as well as an inhibition of the sustained delayed-rectifier voltage-gated $\mathrm{K}^{+}$current and the A-type, fast-inactivating $\mathrm{K}^{+}$current in these neurons. This enhanced sensitivity may be a contributing factor to the pathogenesis of asthma and eosinophilic bronchitis.

\section{Potential anti-inflammatory targets for chronic cough}

\section{Speaker: Kian Fan Chung}

The treatable traits in chronic cough patients are important for clinical treatments. There have been studies demonstrating that eosinophils and their products can increase airway sensory nerve density and sensitize these nerves. IFN- $\gamma$ also enhances the cough reflex sensitivity via Calcium influx in vagal sensory neurons. Hence, neuroinflammatory mechanisms are likely to form the basis for the cough hypersensitivity syndrome, such as direct effect of inflammatory cells on neural tissues. 

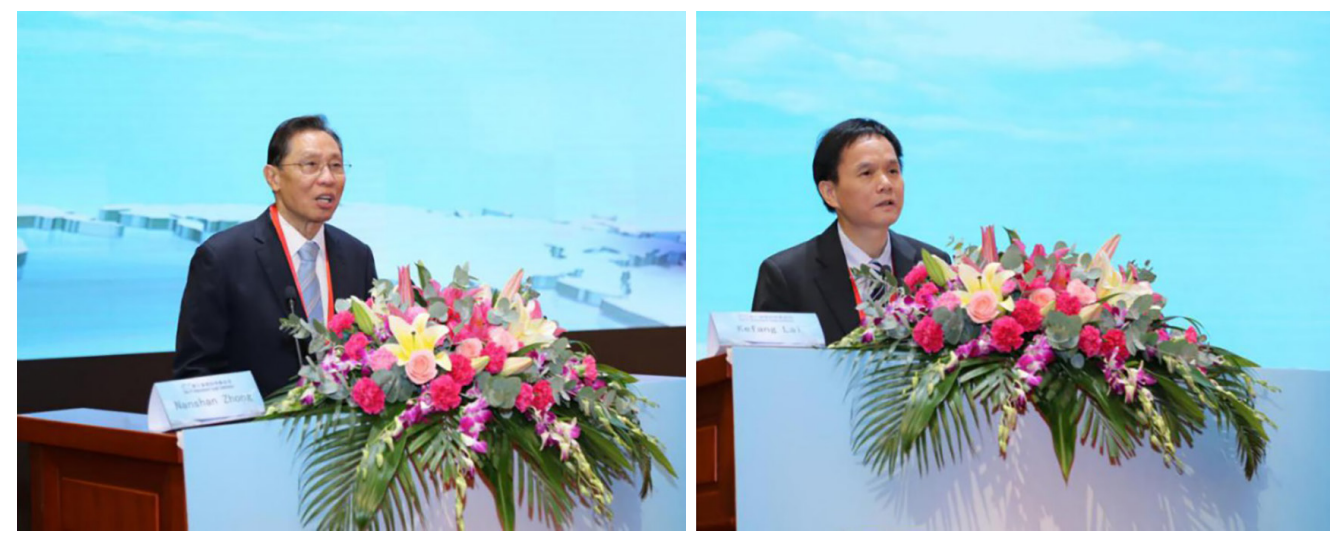

Figure 1 Prof. Nanshan Zhong and Prof. Kefang Lai.

\section{Session 2: Chronic cough and cough hypersensitivity}

\section{Risk factors for chronic cough}

\section{Speaker: Morten Dahl}

Stratified analysis according to different smoking status, with or without asthma/COPD has shown that comorbid asthma/gastroesophageal reflux disease/upper airway cough syndrome, airflow limitation, occupational exposure to dust/ fumes, current smoking and low income are main risk factors of chronic cough in the general population in Denmark. Risk factors for chronic cough may vary with individual and socioeconomic level, smoking status and comorbidity.

\section{Heterogeneity of chronic cough and hypersensitivity}

\section{Speaker: Kefang Lai}

Different clinical phenotypes and the variability in the response to steroid therapies indicate a heterogeneity of chronic cough. Complexity in the peripheral and central neural pathways that regulate cough contributes to the heterogeneity of cough hypersensitivity. Patients with chronic cough exhibit different types of cough hypersensitivity mediated by diverse cough receptors, including TRPV1, TRPA1, citric acid and $\mathrm{PGE}_{2}$. A better understanding of the heterogeneity in cough will provide optimal individualized treatment to patients with refractory chronic cough.

\section{Improving indoor environment for better respiratory bealth: part of precision medicine?}

\section{Speaker: Jim Zhang}

Reduced indoor levels of "outdoor PM2.5" have been found to have a positive impact on adult mortalities. Residential use of air purifier can significantly reduce indoor pollutants concentration and improve airway conditions in children with asthma. True filtration brings a greater improvement in small airway resistance in patients not allergic to dust mite, compared to asthmatics allergic to dust mite. Therefore, precision health should consider allergy status in air pollution interventions and other indoor risk factors.

\section{Chronic cough in Korea}

\section{Speaker: Woo-Jung Song}

Chronic cough affects $2.6 \%$ of general adult population in Korea. The prevalence is higher in older adults and is expected to increase continuously. Features of cough hypersensitivity are commonly observed across age groups. Lack of treatment effects and unclear diagnosis are two major unmet needs of the patients. Upper airway diseases are the most common comorbidity in Korean adult patients. Comorbidity with physician-diagnosed GERD may be one of possible predictors of persistent cough.

\section{Session 3: Evaluation of chronic cough}

\section{Using biomarkers to improve the treatment of chronic cough}

\section{Speaker: Alyn Morice}

Chronic cough is a heterogeneous disease, and cough hypersensitivity is considered as a diagnosis with multiple phenotypes. $\mathrm{P} 2 \mathrm{X} 3$ antagonist is the most promising drug in the treatment of refractory chronic cough in recent years, but antagonizing $\mathrm{P} 2 \mathrm{X} 3$ receptor does not reduce subjects' 


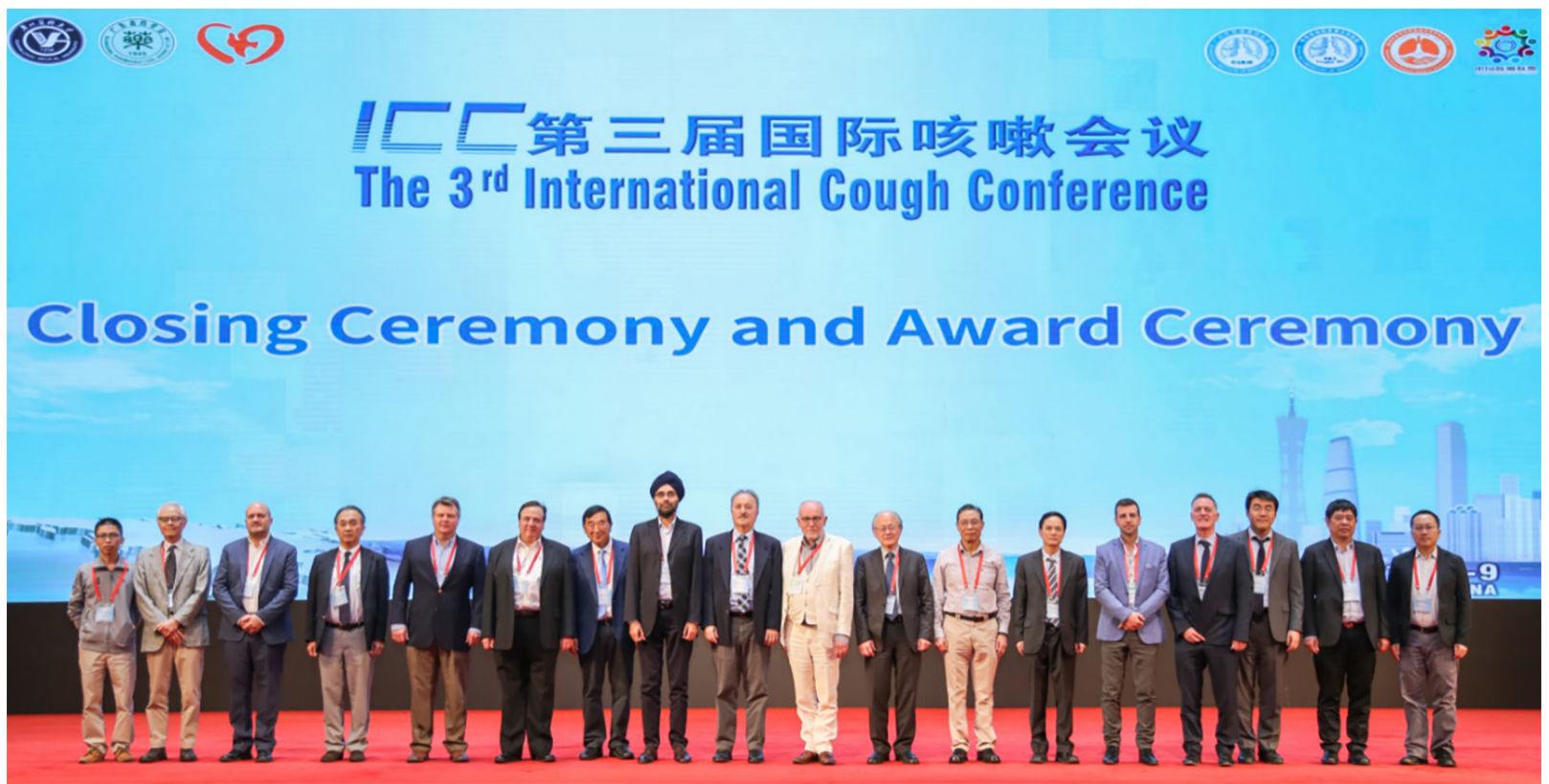

Figure 2 Group photo of experts.
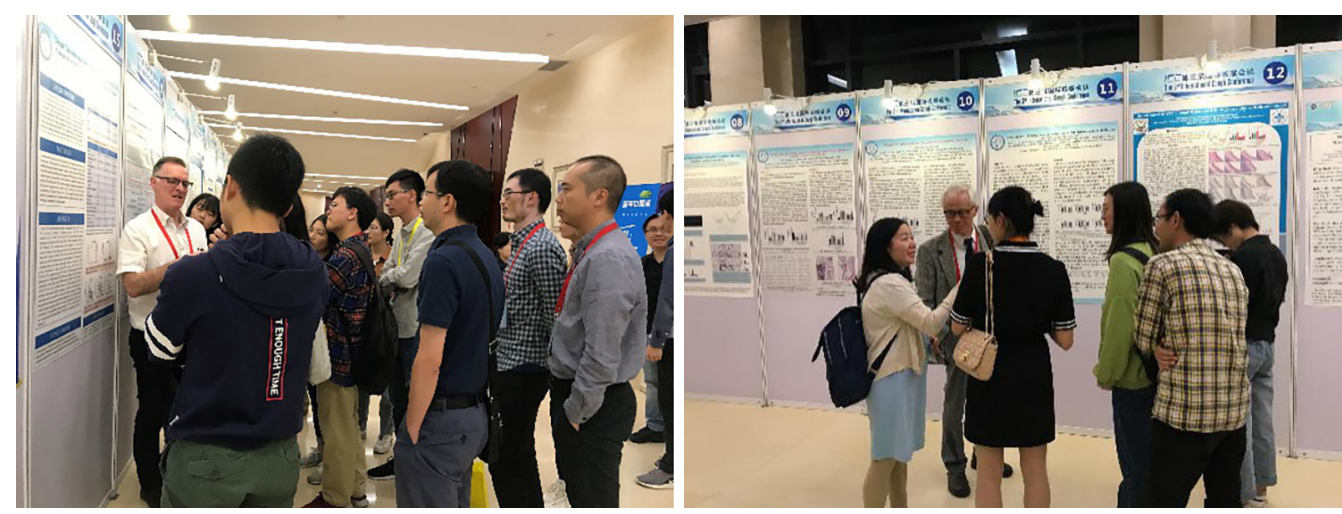

Figure 3 Poster session.

cough sensitivity to capsaicin and citric acid, indicating the heterogeneity of the cough pathway. Identifying different pathways and characteristics of different diseases is critical to the treatment of chronic cough.

\section{Biomarkers of Th2 inflammation and their relevance to cough}

Speaker: Songmin Ying

It is unclear whether eosinophil is a necessary factor for chronic cough, but patients with eosinophilia are sensitive to corticosteroid therapy, therefore, eosinophil inflammation should be carefully evaluated in the management of chronic cough.

\section{Methods for assessing cough sensitivity}

\section{Speaker: Ruchong Chen}

Standardized method of cough provocation test and novel endpoint of cough research are needed. Combining both mechanical and chemical stimuli can comprehensively evaluate cough reflex sensitivity. 


\section{The present and future of cough counting tools}

\section{Speaker: Surinder Birring}

The audio microphone is currently the best tool for monitoring cough, with a certain value in evaluating the efficacy of treatment and predicting the progression of disease. However, cough frequency monitors cannot evaluate indicators such as cough impulse (pharynx itch), throat clearing and cough intensity, and the results of subjects with low cough frequency vary greatly. In the future, high-tech combining indicators such as cough intensity and cough impulse should be used to evaluate cough.

\section{Session 4: Chronic cough in special conditions}

Cough in idiopathic pulmonary fibrosis (IPF)

\section{Speaker: Surinder Birring}

IPF has poor prognosis and unclear etiologies, despite its low prevalence. Around half of ILD-induced cough is due to co-existing diseases, including upper airway cough syndrome, asthma, and gastroesophageal reflux disease. Antifibrotic therapy may have a slight effect on cough suppression. Drugs to treat IPF cough include sodium cromoglicate, thalidomide, $\mathrm{P} 2 \mathrm{X} 3$ antagonist, nalbuphine sustained release tablets and atomized inhaled pirfenidone.

\section{Rare causes of chronic cough}

\section{Speaker: Nanshan Zhong}

Laryngoscope/bronchoscope, pulmonary ventilation and diffusion function, high resolution CT, and PETCT play important roles in the diagnosis of rare causes of chronic cough, including abnormal glottis, laryngeal cancer, enlarged tonsils, OSA, tracheal stenosis, tracheal diverticula, bronchial foreign body, large airway tuberculosis, relapsing polychondritis, pulmonary interstitial fibrosis, lymphangiomyomatosis, sarcoidosis, arrhythmia, esophageal tumor, Hodgkin's lymphoma, and psychogenic cough.

\section{Chronic cough related to special airway eosinophilia}

\section{Speaker: Jiaxing Xie}

Eosinophilic airway inflammation is present in $30-50 \%$ of patients with chronic cough, with common causes including CVA, UACS, and NAEB. Biomarkers of eosinophilic airway inflammation include FeNO and eosinophil, which are mainly mediated by $\mathrm{TH} 2$ cells and ILC2 cells. However, other diseases, such as HES related bone marrow proliferative tumors and IgG4related diseases are also characterized by eosinophilic airway inflammation and chronic cough, which are easily misdiagnosed.

\section{Session 5: Mucus hypersecretion and expectorant}

\section{Management of airway mucus hypersecretion in chronic airway inflammatory disease}

\section{Speaker: Fuqiang Wen}

EGFR pathway is an important signaling pathway to modulate mucus hypersecretion. COPD patients with chronic mucus hypersecretion are more likely to have night-time dyspnea. COPD severity may be associated with high sputum concentration, and $\mathrm{FEV}_{1}$ decline are associated with increased sputum content. Mucoactive drugs could effectively remove phlegm, improve lung function and reduce the incidence of acute exacerbation of COPD.

\section{Mucolytic agents in chronic productive cough}

\section{Speaker: Peter Dicpinigaitis}

There are four classes of mucoactive drugs including mucolytics, mucokinetics, expectorant, and mucoregulators. Mechanisms of mucolytic agents' action poorly understood. Mucolytics may reduce number of exacerbations in chronic bronchitis and COPD. Recent studies have investigated in vitro effects of guaifenesin, while clinical trials demonstrating efficacy was lacking.

\section{Treating cough due to bronchiectasis with non-drug approaches}

\section{Speaker: Lorcan McGarvey}

The most common symptom of bronchiectasis is cough particularly with sputum production. Treatment of respiratory infections and airway clearance techniques are mainstays of management. There is a lack of large and/ or high-quality trials of non-drug techniques of airway clearance, while the absence of high-quality evidence does not imply that efforts to assist airway clearance be 

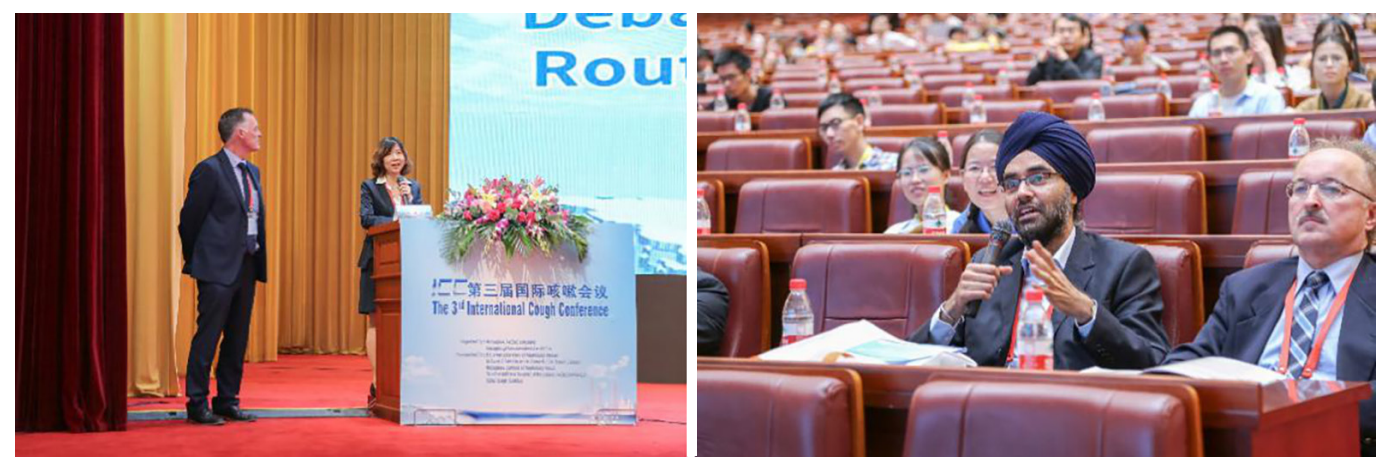

Figure 4 Debate session.

abandoned.

\section{Session 6: Debate: are chest CT and bronchoscopy routine examinations for patients with chronic cough?}

\section{Pro: $\mathrm{Li} \mathrm{Yu}$}

Negative predictive value of Chest $\mathrm{X}$ ray was relatively low in diagnosing pulmonary diseases associated with chronic cough. CT should be the routine investigation for chronic cough since it increases diagnostic accuracy of chough cough without significant adverse effects. Causes of cough changed over time and the proportion of unexplained chronic cough increased. Bronchoscopy is useful in the diagnosis of rare causes of chronic cough.

\section{Con: Lorcan McGarvey}

The diagnostic yield from bronchoscopy in the routine evaluation of chronic cough is low. Investigations are costly, and safety issues need to consider. Current international cough guidelines suggest not to take chest CT scan and bronchoscopy as routine examinations for patients with chronic cough.

\section{Experts' opinion}

$\mathrm{X}$-rays can be routine examinations while CT scan and bronchoscopy can be performed after routine examination fails to diagnose the disease, with personal and local situation taken into account. They cannot serve as a routine examination for chronic cough (Figure 4).

\section{Session 7: Management of chronic cough}

How long should CVA/EB patients be treated?

Speaker: Akio Niimi

Similarities and differences of pathophysiology and natural history were showed in cough variant asthma (CVA), nonasthmatic eosinophilic bronchitis, chronic cough, and classic asthma. Airway hypersensitivity (PC20-Mch), nonuse of inhaled corticosteroids (ICS), allergen exposure and disease duration are risk factors associated with progression of CVA to classic asthma. ICS is the mainstay treatment for CVA, and leukotriene receptor antagonists serve as secondline treatment. Longer-term treatment may be considered in patients with risk factors of developing classic asthma, relapse, chronic airflow obstruction/remodeling.

\section{Treatment of refractory gastroesophageal reflux-induced} chronic cough (GERC): gabapentin vs. baclofen

\section{Speaker: Zhongmin Qiu}

Refractory GERC was mainly caused by insufficient acid suppression, non-acid reflux, transient lower esophageal sphincter relaxations, esophageal hypersensitivity and cough hypersensitivity. Zhongmin Qiu and colleagues found gabapentin have similar therapeutic efficacy to baclofen in treating refractory GERC, but with fewer side effects.

\section{Speech pathology in the management of patients with} chronic refractory cough

\section{Speaker: Gang Wang}

Speech pathology treatment consists of four components including education, cough control techniques, vocal 
hygiene training, and psychoeducational counseling. There were several researches of speech pathology management of chronic refractory cough showing its efficacy of improving cough-related quality of life, and reducing cough reflex sensitivity, urge to cough and cough frequency.

\section{Session 8: New targets for chronic cough}

\section{Challenges for industry in developing novel therapies for chronic cough}

\section{Speaker: Michael Kitt}

The development of new therapies for chronic cough is facing various challenges, including low investment in pharmaceutical companies, critical requirements from regulators on clinical research, and challenges in study design. However, the future of them is bright because the first new drug for chronic cough, gefapixant may be approved in the next few years, which will attract investment in chronic cough treatment.

\section{P2X3 antagonist for refractory chronic cough}

\section{Speaker: Jacky Smith}

P2X3 antagonists are very promising new therapies for refractory and unexplained chronic cough. Gefapixant, a $\mathrm{P} 2 \mathrm{X} 3$ receptors antagonist, can reduce cough frequency and cough severity but with disturbing side effect like dysgeusia. More selective P2X3 antagonists, expected to achieve longterm efficacy, tolerance and safety, are under development.

\section{TRPM8 receptors and cough}

\section{Speaker: Eddie Wei}

TRPM8, a membrane protein serving as an ion channel, can perceive cold, relieve irritation, and raise awareness. In recent years, scholars have been studying its structure and distribution, trying to develop new drugs for chronic cough targeting TRPM8. A new kind of TRPM8 agonist cryosim-3 drops, with ease of use and immediate effects, helps control urge to cough, reduce mucus secretion, and promote effective cough.

\section{Mechanisms of acid-evoked cough}

\section{Speaker: Brendan Canning}

Bicarbonate would enhance acid-induced cough, while acetazolamide, a carbonic anhydrase inhibitor, can suppress it. Brush cells on the airway epithelium may regulate acidinduced cough by carbonic anhydrase and acetylcholine when the concentration of acid in the airway change. The functions of other ion channels and receptors expressed in brush cells need further studies.

\section{Future directions in the treatment of cough}

\section{Speaker: Kian Fan Chung}

Targeting major treatable traits in chronic cough is an important part of treatment flow of chough cough management. Cough hypersensitivity and its heterogeneity were associated with neuro-inflammation and neurosensitivity. The development of new antitussives and neuromodulators would be critical for the treatment of chronic cough. Systems medicine and systems biology should promote precision medicine in cough.

\section{Summary}

The 3rd International Cough Conference was closed successfully. Researchers from China and abroad got to know each other better in this meeting, which will enhance international cooperation on cough field. Let's look forward to the 4th International Cough Conference in 2022.

\section{Acknowledgments}

We would like to thank all speakers for delivering us these excellent speeches and all chairs for presiding the conference. Funding: NSFC 81570092, NSFC 81770098, Incubative Project for Innovation Team of GMU (2017-159) and Clinical Research Foundation of GMU (2017-160).

\section{Footnote}

Conflicts of Interest: The authors have no conflicts of interest to declare.

Ethical Statement: The authors are accountable for all aspects of the work in ensuring that questions related to the accuracy or integrity of any part of the work are appropriately investigated and resolved.

Cite this article as: Luo W, Xu T, Chen Y, Jiang Z, Zhan W, Long L, Lai K. Global specialists talk on latest advances in cough studies: a close-up of the Third International Cough Conference in Guangzhou, China. J Thorac Dis 2019;11(12):57125717. doi: $10.21037 /$ jtd.2019.12.90 